\title{
RSXpSXP Motif
}

National Cancer Institute

\section{Source}

National Cancer Institute. RSXpSXP Motif. NCI Thesaurus. Code C13975.

The 14-3-3 family of proteins mediates signal transduction by binding to phosphoserinecontaining proteins. RSXpSXP Motif is one of two different binding motifs that are present in nearly all known 14-3-3 binding proteins. The14-3-3 dimer binds tightly to single molecules containing tandem repeats of phosphoserine motifs, implicating bidentate association as a signaling mechanism with molecules such as Raf, BAD, and Cbl. (from OMIM) 